\title{
AVALIAÇAO AUDITIVA OBJETIVA ATRAVÉS DE POTENCIAIS EVOCADOS
}

\author{
RICARDO RANIERI SEIXAS *, MARIA IZABEL FASOLO **, \\ ROBERTO NORMÂNDIA MOREIRA ***
}

\begin{abstract}
RESUMO - Analisaram-se 1300 exames de Potencial Evocado Auditivo. Classificaram-se os pacientes segundo faixa de idade, apresentação clínica, relato de fatores de risco que levam a deficiência auditiva e limiar auditivo determinado pelo exame. Distribuíram-se os resultados em 5 faixas de limiar auditivo: audição normal (até 25 dBHL); perđa auditiva moderada (25-50 dBHL); perda acentuada (50-70 dBHL); perda severa (70-90 dBHL); e sem resposta ao estimulo auditivo. Estudaram-se os fatores de risco relativos a gestação, parto e período neonatal, história familiar de deficiência auditiva, malformaçôes do aparelho auditivo, anomalias crânio-faciais, sindromes associadas a deficiência auditiva, certas modalidades de doenças infecciosas e uso de determinadas drogas. Investigaram-se também os itens Retardo do Desenvolvimento Neuropsicomotor, Paralisia Cerebral e os casos sem antecedentes conhecidos. Entre as conclusões destacam-se: $82,8 \%$ dos pacientes eram portadores de alguma forma de deficiência auditiva; o encaminhamento é tardio, evidenciado pelo fato de $54,1 \%$ dos pacientes situarem-se entre 1 e 3 anos de idade; $54,0 \%$ da totalidade dos casos apresentavam-se sem linguagem; 0 fator de risco «Rubéola Congênita» possui a elevada incidéncia de 14,8\%, e este percentual distribuído nas faixas de limiar auditivo revelou um crescimento exponencial, demonstrando inequívoca e acentuada correlação da moléstia com deficiência auditiva.
\end{abstract}

PALAVRAS-CHAVE: potencial evocado auditivo (PEA), audiometria objetiva, audiometria de tronco cerebral, deficiência auditiva.

\section{Hearing impairment evaluation using brain-stem evoled response audiometry}

SUMMARY - We have analyzed 1300 Auditory Brain-Stem Response Audiometry exams. The patients were distributed according to criteria related to age, clinical presentation, hearing impairment risk-factors and hearing thresholds. The results were divided into five groups of hearing thresholds: normal hearing (threshold response obtained up to $25 \mathrm{dBHL}$ ); mild hearing loss (25-50 dBHL); moderate hearing loss (50-70 dBHL); severe hearing loss (70-90 dBHL); and without response to acoustic stimuli. We have studied the risk-factors related to gestation, delivery and neonatal period, family history of hearing loss, hearing apparatus malformations, craniofacial anomalies, certain modalities of infectious diseases, hearing impairment associated syndromes, and the use of some kind of drugs. Cerebral palsy, neuropsychomotor development retardation and cases without known antecedents were also studied. The main results of our study show: $82.8 \%$ incidence of hearing impairment; the late performance of examinations to evidence a definite hearing loss as $54.1 \%$ of the exarrined patients were at ages ranging from one to three years old; $\mathbf{5 4 . 0 \%}$ of tatal cases have not presented any language development; the risk-factor «Congenital Rubeola» has the expressive incidence of $14.8 \%$ and the distribution of this value into hearing threshold ranges has shown an exponential increase which demonstrates a close correlation between that disease and hearing impairment.

KEY WORDS: evoked potentials, brain-stem evoked response audiometry (BERA), hearing impairment.

Trabalho realizado no Servico Integral de Neurofisiologia Clínica, Curitiba: * Médico, Neurofisiologista Clínico; ** Médica, Mestranda em Engenharia Biomédica-CEFET-PR; *** Engenheiro Eletrônico, Mestrando em Engenharia Biomédica - CEFET-PR. Aceite: 6-abril-1993.

Dr. Ricardo Ranieri Seixas - Rua Padre Anchieta 155 - 80410-030 Curitiba PR - Brasil. 
A técnica de Potenciais Evocados vem demonstrando sua acurácia na avaliação da integridade da via auditiva periférica 11. O registro de potenciais evocados permite a determinação objetiva do limiar auditivo, sendo também valiosa ferramenta de auxílio ao diagnóstico de alterações neurológicas através da determinação dos tempos de condução nas fibras auditivas, no seu trajeto até o córtex cerebral. Com a utilização de eletrodos fixados no escalpo, num método não invasivo, estes potenciais podem ser registrados, propiciando um exame simples, preciso, de baixo custo e, em certos casos, com resultados sem comparação com outras técnicas.

O presente estudo foi desenvolvido visando a alcançar dois objetivos: (1) avaliar a casuística referente a deficiência auditiva e (2) relacionar os resultados dos exames de Potencial Evocado Auditivo (PEA) aos vários fatores de risco associados a perda auditiva.

\section{MATHRIAL E METODO}

1. Introducão. A capacidade descritiva, gráfica e analítica do computador torna-o adequado à realização de estudos retrospectivos, principalmente na pesquisa de uma considerável massa de mais de 1000 exames de PEA. Para este estudo, desenvolveu-se um sistema computadorizado dedicado; para a transposição das informaçóes contidas nos prontuários foi elaborado um esquema próprio de classificação e codificação. o limiar auditivo e os fatores de risco que levam à perda auditiva foram as variáveis primárias investigadas em nosso estudo, enquanto a idade, sexo e apresentação clínica, as variáveis auxiliares.

2. Protocolo do Exame. Os procedimentos adotados para os exames seguiram protocolo rigido e uniforme durante o período em que foram realizados; todos os exames foram executados pelo mesmo neurofisiologista e com o mesmo equipamento. $O$ equipamento empregado foi TECA TD-20; eletrodos em forma de disco de $8 \mathrm{~mm}$ de diâmetro eram afixados ao vértex e processog mastóides através de pasta condutiva, obtendo-se uma impedáncia inferior a $5000 \Omega$. Os pacientes, quando necessário e de acordo com idade e peso, receberam dose de hidrato de cloral para induzir o sono e, assim, reduzir a ocorrència de artefatos.

Utilizou-se o método de audiometria por click 5. Esta estimulaçăo auditiva consiste em submeter cada ouvido a clicks com duração de $100 \mu \mathrm{s}$, preferencialmente a uma taxa de repetição de $10 \mathrm{~Hz}$. Com estas características, o click possui componentes harmônicas significativas na faixa de frequência de 500 a $4000 \mathrm{~Hz}$, o que possibilita ampla estimulação da cóclea. No ouvido contralateral, faz-se mascaramento.

Empregou-se tempo de análise de $10 \mathrm{~ms}$, sendo gerados clicks nos modos rarefação, condensação e alternado. Preferencialmente, utiliza-se o modo rarefaçăo 18 . O modo condensação reserva-se aos casos nos quais não seja posisivel obter definição adequada do traçado de ondas gerado pelo modo rarefação, ou naqueles pacientes em que inicialmente não seja detectada a onda V 8. 0 modo alternado emprega-se em níveis de estimulação acima de $80 \mathrm{dBHL}$, a fim de compensar o efeito do artefato produzido pela radiação eletromagnética dos próprios fones de ouvido, sobre a onda I 9. A estimulação atinge o limite máximo de intensidade de 90 dBHL (120 dBSPL).

A avaliação audiométrica através de PEA baseia-se na pesquisa da onda V. Neste procedimento, utilizou-se o método de decréscimo de intensidade do estímulo 15 com passos de 5 dB

Os limiares auditivos obtidos pelos exames foram referidos na escala dBHL.

3. Método de Classificacão. Foram estudados 1300 exames de PEA. Classificaram-se os pacientes segundo a faixa de idade, a apresentação clínica, o relato de fatores de risco que levam a perda auditiva e o limiar auditivo obtido pelo exame. Adotaram-se os seguintes critérios de classificação:

a) Faixa de Idade: 0-3 meses; 3-6 meses; 6-9 meses; 9-12 meses; 1 a 2 anos; 2 a 3 anos;... 9 a 10 anos. Nota: $O$ número total de exames efetuados em pacientes acima de 10 anos é inexpressivo.

\section{b) Apresentação Clínica:}

Anacusia: paciente descrito como sem resposta a qualquer tipo e/ou intensidade de estímulo auditivo;

Hipoacusia: perda parcial da funçăo auditiva; 
Comportamento Auditivo Aleatório: reação inconsistente do paciente aos sons;

Linguagem Restrita: vocabulário reduzido.

Linguagem Rudimentar: poucas palavras, pronunciadas de forma incompleta;

Sem Linguagem;

Retardo na Fala: apresentação diversa do esperado no desenvolvimento normal onde, por volta de 2 anos de idade, a criança expressa-se com palavras simples; em torno de 3 a 4 anos, adquire a forma similar a do adulto;

Regressão na Fala: processo involutivo da linguagem;

Inespecifico: paciente cuja apresentação clínica não se enquadra em qualquer das categorias anteriores.

Cada paciente foi classificado em um desses 9 itens ou numa associação entre eles, conforme a descrição do caso.

c) Fatores de Risco: No total, 22 itens foram pesquisados 2-4,6,7,16,21,22 (Tabela 3). Entre estes, encontram-se os fatores diretamente responsáveis pelo déficit auditivo (19 itens) abrangendo aqueles relativos a gestação, parto e periodo neonatal, história familiar de deficiência auditiva, malformaçōes do aparelho auditivo, anomalias no desenvolvimento do crânio e face, certas modalidades de doenças infecciosas, sindromes associadas a deficiencia auditiva e uso de determinadas drogas. Ainda, incluem-se os quadros passíveis de serem confundidos com déficit auditivo (apresentação clinica sugestiva de déficit auditivo, porém nem sempre ao mesmo relacionada), distribuidos em dois itens: retardo do desenvolvimento neuropsicomotor e paralisia cerebral. Finalmente, temos os casos encaminhados para investigação, porém sem antecedentes conhecidos.

d) Limiar Auditivo: Distribuíram-se os resultados estabelecidos pelo exame em 5 faixas de limiar auditivo: audiçio normal (até 25 dBHL); perda auditiva moderada (25 a 50 dBHL); perda acentuada ( 50 a $\left.70^{\prime} \mathrm{dBHL}\right)$; perda severa (70 a $90 \mathrm{dBHL}$ ) e sem resposta ao estímulo auditivo.

\section{RESLITADOS E COMENTARIOS}

Constatou-se ligeira predominância de pacientes do sexo masculino, havendo $720 \quad(55,4 \%)$ pacientes do sexo masculino e $580(44,6 \%)$ do sexo feminino. A distribuição da idade da população pode ser vista na Figura 1. Nota-se que entre 1 e 3 anos de idade estão concentrados $54,1 \%$ dos pacientes. A média de idade da população situou-se na faixa de 2 a 3 anos.

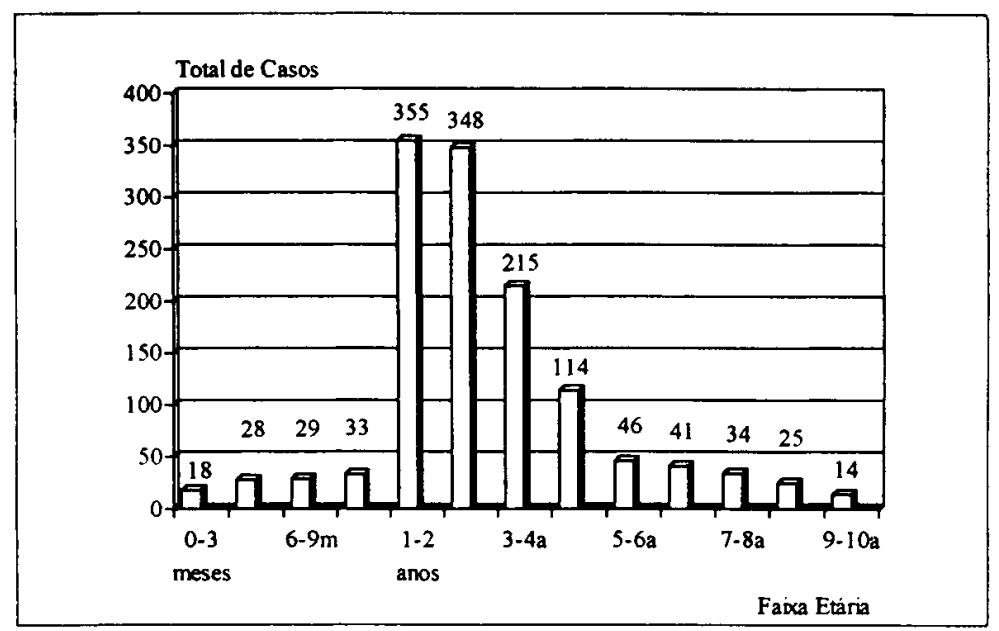

Fig. I Distribuiçâo segumdo a idade da população esfudada. 
Na Figura 2 temos a distribuição dos pacientes nas faixas de limiar auditivo. Observa-se um comportamento similar para ambos os ouvidos. Na população pesquisada, temos que, bilateralmente, apenas $17,2 \%$ (224 casos) possuiam audição normal, enquanto $27,1 \%$ (352 casos) não apresentavam resposta ao estímulo auditivo.

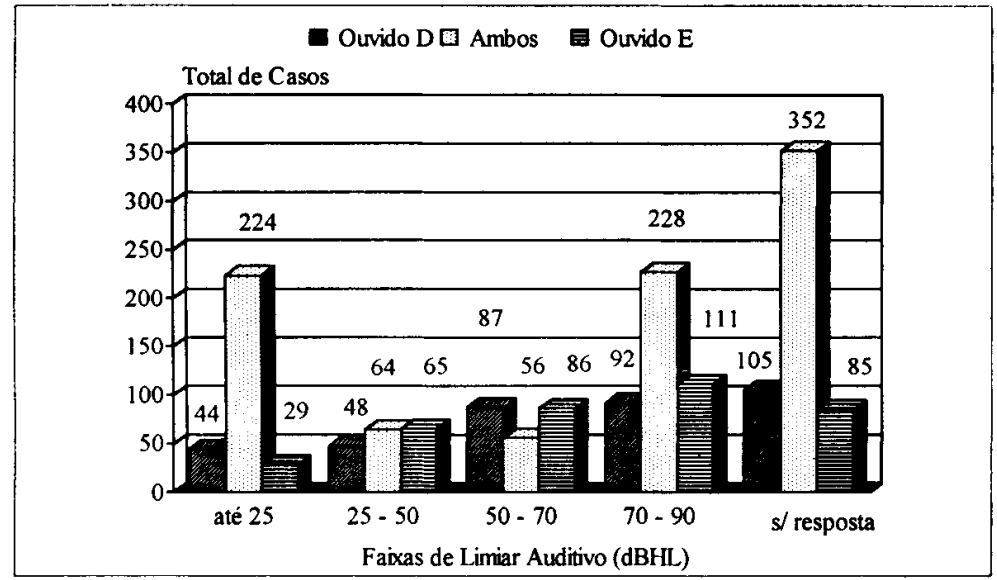

lïg 2. Distribuição nas faixas de limiar anditivo.

Os dados estatísticos relativos a apresentacão clínica são vistos na Tabela 1. Nota-se incidência de $54,0 \%$ do total dos pacientes descritos, como sem linguagem. De modo geral, há uma distribuição coerente relativa à proporção dos sexos na população estudada; entretanto, as apresentações de retardo na fala e linguagem rudimentar mostram-se com maior incidência para o sexo masculino (respectivamente, $69,5 \%$ e $60,5 \%$ ). Nota-se ainda que os pacientes referidos como anacúsicos, apesar da distribuição geral da população por sexo ter predominância masculina, atingiram uma distribuição equivalente para ambos os sexos (50,1\% para masculino e $49,9 \%$ para feminino).

Estudamos o comprometimento de cada ouvido individualmente, ressaltando-se os casos em que ambos os ouvidos encontram-se na mesma faixa de limiar. Nas Tabelas 2, 4, 5 e 6, cada faixa de limiar auditivo possui três colunas: a primeira coluna (OD) corresponde ao número de pacientes cujo ouvido direito, apenas, encontra-se naquela faixa, enquanto o ouvido esquerdo destes mesmos pacientes situa-se em outra faixa qualquer. A segunda coluna (\&) corresponde ao número de pacientes nos quais ambos os ouvidos situam-se naquela mesma faixa de limiar. A terceira coluna (OE) refere-se ao ouvido esquerdo de forma análoga à primeira coluna.

O cruzamento dos dados da apresentação clínica com os limiares auditivos dos exames sāo apresentados na Tabela 2 . Observa-se que 4,1\% (16 casos) dos pacientes referidos como

Tabela 1. Classificação segundo a apresentação clínica.

\begin{tabular}{|c|c|c|c|c|c|c|}
\hline \multirow[b]{2}{*}{ Apresentação clínica } & \multicolumn{2}{|c|}{ Total } & \multicolumn{2}{|c|}{ Masculino } & \multicolumn{2}{|c|}{ Feminino } \\
\hline & $\mathbf{n}$ & $\%$ & $\mathbf{n}$ & $\%$ & $\mathrm{n}$ & $\%$ \\
\hline Anacusia & 391 & 30,1 & 196 & $\mathbf{5 0 , 1}$ & 195 & 49,9 \\
\hline Hipoacusia & 316 & 24,3 & 187 & 59,2 & 129 & 40,8 \\
\hline Comportamento auditivo aleatório & 234 & 18,0 & 124 & 53,0 & 110 & 47,0 \\
\hline Sem linguagem & 702 & 54,0 & 379 & 54,0 & 323 & 46,0 \\
\hline Linguagem rudimentar & 114 & 8,8 & 69 & 60,5 & 45 & 39,5 \\
\hline Linguagem restrita & 49 & 3,8 & $\mathbf{2 9}$ & 59,2 & 20 & 40,8 \\
\hline Retardo na fala & 105 & 8,1 & 73 & 69,5 & 32 & 30,5 \\
\hline Regressão na fala & 42 & 3,2 & 23 & 54,8 & 19 & 45,2 \\
\hline Inespecifico & 133 & 10,2 & 82 & 61,7 & 51 & 38,3 \\
\hline
\end{tabular}




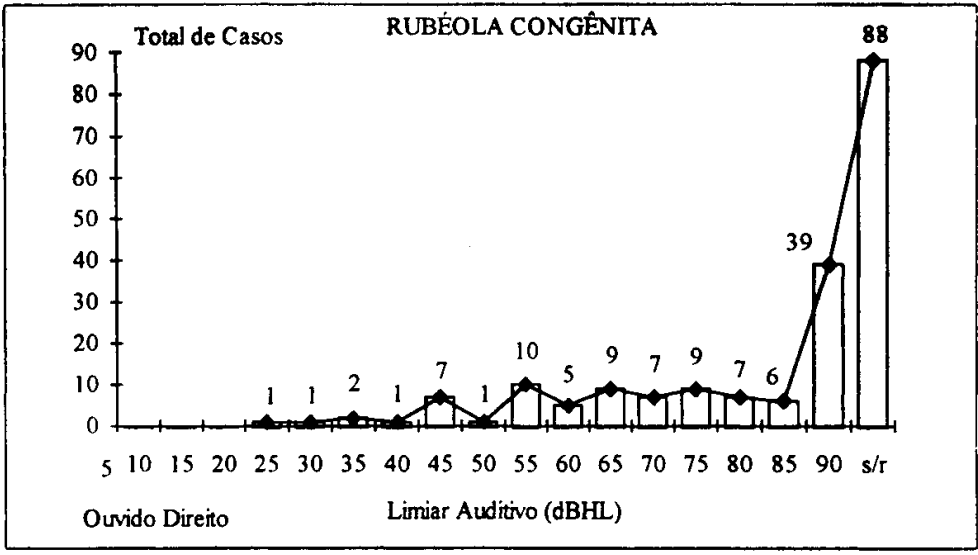

Fig. 3 Rubéola Congênita: distribuição nas faixas de limiar auditivo. s/r, sem resposta.

Tabela 2. Apresentação clínica $\times$ limiar anditivo (dBHL).

\begin{tabular}{|c|c|c|c|c|c|c|c|c|c|c|c|c|c|c|c|}
\hline \multirow[b]{2}{*}{$\mathbf{A C}$} & \multicolumn{3}{|c|}{ até 25} & \multicolumn{3}{|c|}{25 a 50} & \multicolumn{3}{|c|}{50 a 70} & \multicolumn{3}{|c|}{70 a 90} & \multicolumn{3}{|c|}{ sem resposta } \\
\hline & OD & $\&$ & $\mathrm{OE}$ & $\mathrm{OD}$ & $\&$ & $\mathrm{OE}$ & OD & $\&$ & $\mathrm{OE}$ & OD & $\&$ & OE & OD & $\&$ & OF \\
\hline $\mathbf{A}$ & 7 & 16 & 2 & 5 & 3 & 4 & 15 & 6 & 11 & 24 & 80 & 39 & 36 & 199 & 31 \\
\hline $\mathbf{H}$ & 6 & 17 & 13 & 19 & $\mathbf{1 5}$ & 20 & 40 & 22 & 42 & 37 & 61 & 31 & 32 & 67 & 28 \\
\hline CA & 8 & 51 & - & 4 & 13 & 17 & 17 & 9 & 13 & 10 & 58 & 22 & 22 & 42 & 9 \\
\hline SL & 16 & 105 & $\mathbf{5}$ & 21 & 13 & 24 & 42 & 19 & 44 & 52 & 138 & 63 & 61 & 235 & 56 \\
\hline LR & 2 & 24 & 4 & 8 & 8 & 9 & 7 & 13 & 11 & 10 & 21 & 6 & 8 & 13 & 5 \\
\hline LI & 2 & 13 & 1 & 2 & 2 & 7 & 8 & $\mathbf{5}$ & 2 & 1 & 7 & 7 & 7 & 2 & 3 \\
\hline RF & 5 & 36 & 3 & 6 & 15 & 9 & 8 & 11 & 5 & 1 & 8 & 6 & 6 & 9 & 3 \\
\hline $\mathbf{R E}$ & 1 & 6 & 2 & 4 & 1 & 1 & 1 & 1 & 3 & 2 & 8 & 3 & 3 & 15 & 2 \\
\hline I & 9 & 42 & 6 & 5 & 17 & 9 & 9 & 6 & 6 & 10 & 10 & 10 & 6 & 19 & 8 \\
\hline
\end{tabular}

AC, apresentação clínica; OD, ouvido direito; \&, ambos os ouvidos; OE, ouvido esquerdo; A, anacusia; H, hipoacusia; CA, comportamento auditivo aleatório; SL, sem linguagem; LR, linguagem rudimentar; LI, linguagem restrita; $R F$, retardo na fala; RE, regressão na fala; I, inespecífico.

anacúsicos e 5,4\% (17 casos) como hipoacúsicos encontravam-se na faixa de limiar auditivo normal, bilateraimente. Ainda, temos que 15,0\% (105 casos) dos pacientes descritos como sem linguagem apresentavam-se, da mesma forma, com limiares auditivos bilateralmente normais.

Na Tabela 3 temos os dados relativos aos fatores de risco. Os fatores de maior incidência são: rubéola $(14,8 \%)$, otite $(8,7 \%)$, meningoencefalite $(6,0 \%)$, e história familiar de deficiência auditiva $(6,0 \%)$. Observa-se também uma incidência expressiva de retardo no desenvolvimento neuropsicomotor $(17,0 \%)$. Além disto, nota-se que Rubéola, apesar da predominância do sexo masculino na população estudada, mostra distribuição equivalente para ambos os sexos (50,8\% para sexo masculino e $49,2 \%$ para sexo feminino); ao passo que otite apresenta uma distribuicão predominante para o sexo masculino $(60,2 \%)$.

Na Tabela 4 temos a análise da totalidade dos casos classificados segundo os fatores de risco e distribuidos nas faixas de limiar auditivo. $O$ fator Retardo no desenvolvimento neuropsicomotor apresenta-se, em 38,0\% (84 pacientes) dos casos, com limiar auditivo normal, bilateralmente. Nos fatores de maior incidência, vemos também os pacientes com audição normal nas seguintes percentagens: Otite 6,2\% (7 casos); Meningoencefalite 5,1\% (4 casos); História familiar de deficiencia auditiva 5,1\% (4 casos). Entretanto, em todos os casos relativos ao fator Rubéola constatou-se algum grau de perda auditiva (somente 1 caso com ouvido direito no limiar normal, porém ouvido esquerdo com perda). 
Tabela 3. Fatores de risco pesquisados: distribuição por sexo.

\begin{tabular}{|c|c|c|c|c|c|c|}
\hline \multirow[b]{2}{*}{ Fator de risco } & \multicolumn{2}{|c|}{ Total } & \multicolumn{2}{|c|}{ Masculino } & \multicolumn{2}{|c|}{ Feminino } \\
\hline & $\mathrm{n}$ & $\%$ & $\mathbf{n}$ & $\%$ & $\mathrm{n}$ & $\%$ \\
\hline Rubéola * & 193 & 14,8 & 98 & 50,8 & 95 & 49,2 \\
\hline Otite & 113 & 8,7 & 68 & 60,2 & 45 & 39,8 \\
\hline Meningoencefalite & 78 & 6,0 & 38 & 48,7 & 40 & $\mathbf{5 1 , 3}$ \\
\hline História familiar de deficiência auditiva & 78 & 6,0 & 43 & 55,1 & 35 & 44,9 \\
\hline Malformações do aparelho auditivo & 17 & 1,3 & 11 & 64,7 & 6 & $\mathbf{3 5 , 3}$ \\
\hline Pré-natal & 20 & 1,5 & $\mathbf{9}$ & 45,0 & 11 & 55,0 \\
\hline Parto & 69 & 5,3 & 43 & 62,3 & 26 & 37,7 \\
\hline Asfixia perinatal & 9 & 0,7 & 7 & 77,8 & 2 & 22,2 \\
\hline Prematuridade & 71 & 5,5 & 44 & 62,0 & 27 & 38,0 \\
\hline Peso $\leqslant 1500 \mathrm{~g}$ & 11 & 0,8 & 7 & 63,6 & 4 & 36,4 \\
\hline Hiperbilirrubinemia & 25 & 1,9 & 14 & 56,0 & 11 & 44,0 \\
\hline Periodo neonatal & 46 & 3,5 & 34 & 73,9 & 12 & 26,1 \\
\hline Incubadora & 24 & 1,8 & 16 & 66,7 & 8 & 33,3 \\
\hline Anomalias crânio-faciais & 29 & 2,2 & 19 & 65,5 & 10 & 34,5 \\
\hline Antibiótico & 42 & 3,2 & 26 & 61,9 & 16 & 38,1 \\
\hline Sindromes associadas & 3 & 0,2 & - & - & 3 & 100 \\
\hline Toxoplasmose * & 7 & 0,5 & 4 & 57,1 & 3 & 42,9 \\
\hline Citomegalovirus * & 1 & 0,1 & 1 & 100 & - & - \\
\hline Sifilis * & 2 & 0,2 & 1 & 50,0 & 1 & 50,0 \\
\hline Paralisia cerebral & 37 & 2,8 & 15 & 40,5 & 22 & $\mathbf{5 9 , 5}$ \\
\hline Retardo no desenv. neuropsicomator & 221 & 17,0 & 119 & 53,8 & 102 & 46,2 \\
\hline Sem antecedentes conhecidos & 421 & 32,4 & 230 & 54,6 & 191 & 45,4 \\
\hline
\end{tabular}

* infeccão congênita.

Tabela 4. Fatores de risco por faixa de limiar auditivo (dBHL).

\begin{tabular}{|c|c|c|c|c|c|c|c|c|c|c|c|c|c|c|c|}
\hline \multirow[b]{2}{*}{ FR } & \multicolumn{3}{|c|}{ até 25} & \multicolumn{3}{|c|}{25 a 50} & \multicolumn{3}{|c|}{50 a 70} & \multicolumn{3}{|c|}{70 a 90} & \multicolumn{3}{|c|}{ sem resposta } \\
\hline & OD & $\&$ & $\mathrm{OE}$ & $\mathrm{OD}$ & $\&$ & OE & OD & $\&$ & OE & OD & $\&$ & $\mathrm{OE}$ & OD & $\&$ & OE \\
\hline $\mathbf{R u} *$ & 1 & 一 & - & 7 & $\mathbf{5}$ & 9 & 21 & 10 & 18 & 15 & 46 & 25 & 25 & 63 & 17 \\
\hline ot & 4 & 7 & 3 & $\mathbf{5}$ & 2 & 10 & 14 & 8 & 6 & 6 & 25 & 15 & 11 & 31 & 6 \\
\hline $\mathrm{Me}$ & 4 & 4 & 3 & 3 & 3 & $\mathbf{5}$ & 5 & 1 & 3 & $\mathbf{5}$ & 11 & 8 & 8 & 34 & 6 \\
\hline $\mathbf{H F}$ & - & 4 & - & 1 & 2 & 1 & 6 & 2 & 5 & 10 & 18 & 9 & 7 & 28 & 9 \\
\hline Mf & 3 & - & 4 & 1 & 1 & 3 & 2 & 1 & 一 & 3 & 4 & 3 & $\mathbf{2}$ & - & 1 \\
\hline Pr & 5 & 12 & - & 5 & 3 & 4 & 3 & 3 & 6 & 3 & 15 & 5 & 2 & 20 & 3 \\
\hline PN & 2 & 3 & - & 2 & - & 2 & 2 & $\longrightarrow$ & 2 & 1 & 1. & 3 & 3 & 6 & 3 \\
\hline $\mathrm{Pa}$ & 2 & 26 & 1 & 4 & - & 3 & 7 & 1 & 3 & 3 & 5 & 6 & 3 & 18 & 6 \\
\hline $\mathbf{A} \mathbf{T}$ & - & 2 & - & 1 & 1 & 1 & 2 & 4 & - & 1 & 9 & 4 & 2 & 20 & $\mathbf{1}$ \\
\hline CF & 3 & 6 & - & 1 & 5 & 2 & - & - & 2 & - & 5 & - & - & $\mathbf{9}$ & - \\
\hline lc & 1 & 5 & - & 2 & - & 2 & 3 & 1 & 3 & 3 & 2 & 2 & - & 7 & 2 \\
\hline $\mathbf{B i}$ & - & 5 & - & - & 1 & - & 1 & - & - & 1 & 6 & - & - & 11 & 2 \\
\hline RN & 6 & 6 & - & 2 & 3 & 6 & 8 & 2 & $\mathbf{1}$ & - & 7 & 7 & 2 & 10 & 4 \\
\hline $\mathrm{Pe}$ & 1 & 1 & - & - & - & 1 & 1 & 1 & 1 & 1 & 2 & 1 & - & 4 & 1 \\
\hline Af & - & 2 & - & 1 & - & 一 & - & - & 1 & - & 4 & 一 & - & 2 & - \\
\hline Sd & - & - & - & - & - & - & - & - & 1 & 1 & - & - & - & 2 & - \\
\hline $\mathbf{T x} *$ & 1 & - & - & - & 2 & 1 & 1 & - & 1 & 1 & 2 & 1 & - & - & - \\
\hline $\mathrm{Cm}$ & - & 1 & - & - & - & - & - & - & - & $\rightarrow$ & - & - & - & - & - \\
\hline $\mathbf{S i} *$ & - & - & - & - & - & - & - & 1 & 1 & 1 & - & - & - & - & - \\
\hline $\mathrm{PC}$ & 2 & 9 & 1 & 2 & 4 & 2 & 一 & - & 3 & 4 & 5 & 1 & 1 & 10 & 2 \\
\hline $\mathrm{RD}$ & 9 & 84 & 6 & 6 & 9 & 12 & 17 & 4 & 6 & 7 & 21 & 11 & 6 & 58 & 10 \\
\hline $\mathbf{S A}$ & 15 & 88 & 10 & 17 & 27 & 16 & 16 & 21 & 34 & 31 & 72 & 30 & 34 & 100 & 23 \\
\hline
\end{tabular}

FR, fatores de risco; OD, ouvido direito; \&, ambos os ouvidos; OE, ouvido esquerdo; Ru, rubéola; Ot, otite; Me, meningoencefalite; HF, história familiar de deficiência; Mf, malformações do aparelho auditivo; Pr, prematuridade; PN, pré-natal; Pa, parto; AT, antibiótico; CF, anomalias cranio-faciais; Ic, incubadora; $B 1$, hiperbilirrubinemia; $R N$, periodo neonatal; Pe, peso $\leqslant 1500 \mathrm{~g}$; Af, asfixia perinatal; Sd, sindromes associadas; $T \mathrm{x}$, toxoplasmose; Cm, citomegalovírus; Si, sifllis; PC, paralisia cerebral; RD, retardo do desenvolvimento neuropsicomotor; SA, sem antecedentes conhecidos; *, infecçăo congênita. 
Tabela 5. Limiar auditivo (dBHL) relativo à fase da gestação na qual ocorreu rubéola.

\begin{tabular}{|c|c|c|c|c|c|c|c|c|c|c|c|c|c|c|c|}
\hline \multirow[b]{2}{*}{$T G$} & \multicolumn{3}{|c|}{ atê 25} & \multicolumn{3}{|c|}{25 a 50} & \multicolumn{3}{|c|}{50 a 70} & \multicolumn{3}{|c|}{70 a 90} & \multirow{2}{*}{$\begin{array}{l}\text { sem } \\
\text { OD }\end{array}$} & \multicolumn{2}{|c|}{ resposta } \\
\hline & OD & $\&$ & OE & OD & $\&$ & $\mathrm{OE}$ & OD & $\&$ & $\mathrm{OE}$ & OD & $\&$ & $\mathrm{OE}$ & & $\&$ & $\mathrm{OE}$ \\
\hline $1 \mathrm{~T}$ & 1 & - & - & 6 & 3 & 6 & 15 & 6 & 14 & 11 & 35 & 17 & 17 & 39 & 13 \\
\hline $2 \mathrm{~T}$ & - & 一 & - & 1 & - & 1 & 一 & 2 & 3 & 3 & 6 & 3 & 3 & 8 & 一 \\
\hline $3 T$ & 一 & - & - & - & 1 & - & - & 一 & 1 & 1 & - & - & - & 2 & - \\
\hline
\end{tabular}

$T G$, trimestre gestacional; $O D$, ouvido direito; \&, ambos os ouvidos; OE, ouvido esquerdo; 1T, primeiro trimestre; $2 T$, segundo trimestre; $3 T$, terceiro trimestre.

Tabela 6. Limiar auditivo (dBHL) estabelecido nos casos relacionados à suspeita de rubéola durante a gestação.

\begin{tabular}{|c|c|c|c|c|c|c|c|c|c|c|c|c|c|c|c|}
\hline \multicolumn{3}{|c|}{ até 25} & \multicolumn{3}{|c|}{25 a 50} & \multicolumn{3}{|c|}{50 a 70} & \multicolumn{3}{|c|}{70 a 90} & \multicolumn{3}{|c|}{ sem resposta } & \multirow[b]{2}{*}{ Total } \\
\hline OD & $\&$ & $\mathrm{OE}$ & OD & $\&$ & $\mathrm{OE}$ & OD & $\&$ & OE & OD & $\&$ & $\mathrm{OE}$ & OD & $\&$ & $\mathrm{OE}$ & \\
\hline 一 & 1 & - & 1 & 2 & 1 & 3 & 1 & - & 1 & 6 & 7 & 5 & 11 & 2 & 31 \\
\hline
\end{tabular}

OD, ouvido direito; \&, ambos os ouvidos; OE, ouvido esquerdo.

Aprofundando-se a investigação do fator Rubéola, foi analisado o parâmetro fase gestacional (trimestre) do acometimento, referido em 160 casos. No primeiro trimestre, relatam-se 133 casos; no segundo, 23 casos; a no terceiro trimestre, há 4 casos. Os resultados encontram-se resumidos na Tabela 5.

Na Tabela 6 temos os casos associados à suspeita de Rubéola congênita. A mãe descreve moléstia eruptiva febril, branda, de rápida evolução, durante a gravidez, cujo diagnóstico de rubéola não foi estabelecido. Não obstante, a descrição é sugestiva, e a mãe em geral relata nunca haver tido episódios alérgicos cutâneos. Os resultados obtidos através do PEA corroboram a hipótese, sendo plenamente compatíveis àqueles de rubéola confirmada.

\section{CONCLUSÕES}

Constatou-se elevada incidência $(82,8 \%$ dos casos) de pacientes com alguma forma de deficiência auditiva.

Vimos que $54,1 \%$ dos pacientes submetidos ao PEA encontravam-se entre 1 e 3 anos de idade. Sabendo-se que a deficiência auditiva tem origem principalmente nas fases pré-natal, perinatal e neonatal, é tardia a realização deste exame conclusivo de deficiência auditiva.

$\mathrm{Na}$ população em estudo, verificou-se uma incidência de $54,0 \%$ de casos referidos como sem linguagem. Destes pacientes, $15,0 \%$ encontravam-se na faixa de limiar auditivo normal, bilateralmente, sendo os restantes $85,0 \%$ portadores de algum grau de deficiência auditiva. Considerando-se que a deficiência auditiva prejudica o desenvolvimento da criança, principalmente limitando ou impedindo a aquisição da linguagem e da fala, por conseguinte dificultando-lhe a integração na família e no ambiente social, é imperativo que se estabeleça um programa de "follow-up» com medidas de avaliação objetiva da audição desde o nascimento $1,7,10,14,17,19,20$.

Constatou-se também que $4,1 \%$ dos pacientes descritos como anacúsicos e $5,4 \%$ como hipoacúsicos encontravam-se na faixa de limiar auditivo normal, bilateralmente. Assim, vemos que a determinação precisa do limiar auditivo através do registro de potenciais evocados viabiliza o diagnóstico acerca da presença ou não de deficiência auditiva, possibilitando o acompanhamento adequado dos pacientes. 
O fator de risco Rubéola congênita possui uma incidência elevada $(14,8 \%)$. Este percentual, distribuído em limiar auditivo a intervalos de $5 \mathrm{~dB}$ (fig. 3 ), revelou um crescimento exponencial (o número de casos aumenta expressivamente nas faixas de maior perda auditiva). Tal comportamento demonstra inequívoca e acentuada correlação com deficiência auditiva, independente da fase do ciclo gestacional. Considerando-se que a rubéola é doença infecto-contagiosa de caráter viral de controle através da vacinação e que, no Brasil, este procedimento é opcional, sugere-se fortemente sua introdução num programa regular de vacinação $12,13,23,24$.

\section{REFERENCIAS}

1. Adelman C, Levi H, Linder N, Sohmer H. Neonatal auditory brain-stem response threshold and latency: 1 hour to 5 months. Electroenceph Clin Neurophysiol 1990, 77:77-80.

2. American Academy of Pediatrics. Joint Committee on Infant Hearing Position Statement 1982. Pediatrics 1982, 70:496-497.

3. Barden TP, Peltzman P. Newborn brain stem auditory evoked responses and perinatal clinical events. Am J Obstetr Gynecol 1980, 136:912-919.

4. Be.s FH, Peek BF, Chapman JJ. Further observations on noise levels in infant incubators. Pediatrics 1979, 63:100-106.

5. Chiappa KH. Evoked potentials in clinical medicine. Ed 2. New York: Raven Press, 1990.

6. Douek E, Dodson HC, Banister LH, et al. Effects of incubator noise on the cochlea of the newborn. Lancet 1976, 2:1110.

7. Duara S, Suter MS, Bessard KK, Gutberlet RL. Neonatal screening with auditory brainstem responses: results of follow-up audiometry and risk factor evaluation. J Pediatr 1986, $168: 276$.

8. Emerson RG, Brooks EB, Parker SW, Chiappa KH. Effects of click polarity on brainstem auditory evoked potentials in normal subjects and patients: unexpected sensitivity of wave V. Ann NY Acad Sci 1982, 388:710-721.

9. Epstein CM. The use of brain stem auditory evoked potentials in the evaluation of the central nervous system. Neurologic Clinics 1988, 6:771-790.

10. Feinmesser M, Tell L, Levi H. Follow-up of 40,000 ifants screened for hearing defect. Audiology 1982, 21:197.

11. Goidie $W$. The brainstem auditory evoked potentiais in infants and children. $J$ Clin Neurophysiol 1992, 9:394-407.

12. Goodwin $\mathbf{S}$. Preventive care for children: immunization in England and Wales. Pediatrics $1 \subseteq$ C0, 86(Suppl) : 1056-1060.

13. Hinman A. Immunizations in the United States. Pediatrics 1990, 86(Suppl):1064-1066.

14. Lary S, Briassoulis G, Vries L, Dubowitz L, Dubowitz V. Hearing threshold in preterm and term infants by auditory brainstem response. $J$ Pediatr 1985, 107:593-599.

15. Legat A, Arezzo J, Vaughan H Jr. The anatomic and physiologic bases of brainstem auditory evoked potentials. Neurologic Clinics 1988, 4:681-704.

16. Picton $T$, Durieux-Smith A. Auditory evoked potentials in the assessment of hearing. Neurologic Clinics 1988, 4:791-808.

17. Schulman-Galambos C, Galambos R. Brain stem evoked response audiometry in newborn hearing screening. Arch Otolaryngol 1979, 105:86-90.

18. Schwartz DM, Morris MD, Spydell JD, Brink CT, Grim MA, Schwartz JA. Influence of click polarity on the brain-stem auditory evoked response (BAER) revisited. Electroenceph Clin Neurophysiol 1988, 77:445-457.

19. Shannon D, Felix J, Krumhoz A, Goldstein P, Harris $K$. Hearing screening of high-risk newborns with brainstem auditory evoked potentials: a follow-up study. Pediatrics 1984, $73: 22-26$.

20. Starr A, Amiie RN, Martin WH, Sanders S. Development of auditory function in newborn infants revealed by auditory brain-stem potentials. Pediatrics 1977, 60:831-839.

21. Stein LK, Kraus $N$, Ozdamar $O$ et al. Hearing loss in an institutionalized mentally retarded population: identification by auditory brainstem response. Arch Otolaryngol Head Neck Surg 1987, 113:32-35.

22. Suzuki J, Kodera $\mathbf{K}$, Kaga $\mathbf{K}$. Auditory evoked brainstem response assessment in otolaryngology. Ann NY Acad Sci 1982, 388:487-500.

23. Verbrugge $H$. The national immunization program of the Netherlands. Pediatrics 1990, 86(Suppl):1060-1063.

24. Verbrugge H. Children with special needs in the Netherlands: impaired hearing, adolegcent pregnancy, and myelomeningocele. Pediatrics 1990, 86(Suppl):1117-1119. 